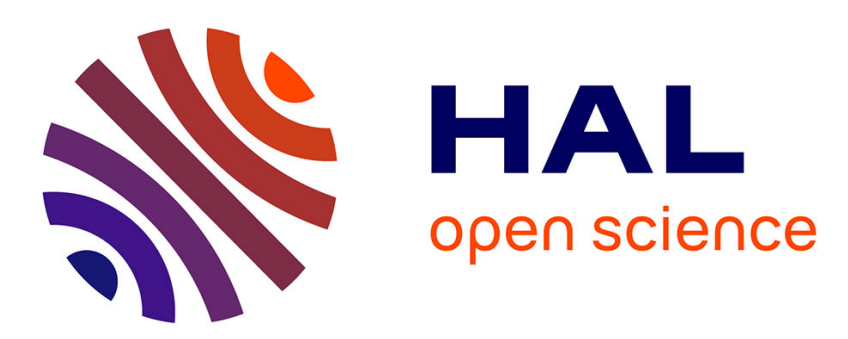

\title{
Surveillance et contrôle de la pollution nitrique agricole en Terrefort aquitain
}

\author{
Martin Paegelow
}

\section{To cite this version:}

Martin Paegelow. Surveillance et contrôle de la pollution nitrique agricole en Terrefort aquitain. Revue Géographique des Pyrénées et du Sud-Ouest, 1991, 62 (1), pp.89-95. 10.3406/rgpso.1991.3240 . hal02866018

\section{HAL Id: hal-02866018 https://hal.science/hal-02866018}

Submitted on 12 Jun 2020

HAL is a multi-disciplinary open access archive for the deposit and dissemination of scientific research documents, whether they are published or not. The documents may come from teaching and research institutions in France or abroad, or from public or private research centers.
L'archive ouverte pluridisciplinaire HAL, est destinée au dépôt et à la diffusion de documents scientifiques de niveau recherche, publiés ou non, émanant des établissements d'enseignement et de recherche français ou étrangers, des laboratoires publics ou privés. 


\section{Surveillance et contrôle de la pollution nitrique agricole en Terrefort} aquitain

\section{Martin Paegelow}

\section{Citer ce document / Cite this document :}

Paegelow Martin. Surveillance et contrôle de la pollution nitrique agricole en Terrefort aquitain. In: Revue géographique des Pyrénées et du Sud-Ouest, tome 62, fascicule 1, 1991. pp. 89-95;

doi : https://doi.org/10.3406/rgpso.1991.3240

https://www.persee.fr/doc/rgpso_0035-3221_1991_num_62_1_3240

Fichier pdf généré le 06/04/2018 
REVUE GÉographique des PYRÉNÉES ET DU SUD-OUEST TOME 62, FASC. 1, pp. 89-99, Toulouse, 1991.

\title{
CHRONIQUE
}

\author{
SURVEILLANCE ET CONTROLE \\ DE LA POLLUTION NITRIQUE AGRICOLE \\ EN DOMAINE DE TERREFORT AQUITAIN
}

Les gains de productivité considérab!es qui ont marqué l'agriculture européenne depuis une quarantaine d'années, sont dus, entre autres, à l'emploi croissant d'engrais azotés. Les effets indésirables de l'augmentation des apports azotés, dont une part importante est transférée principalement sous forme de nitrate - vers les nappes souterraines et les cours d'eau, se sont manifestés depuis le début des années 1980 par une rapide dégradation de la qualité des eaux. Parallèlement, les recherches aussi bien fondamentales que celles destinées à contenir la pollution nitrique se sont multipliées sur la question (1).

En 1980, une norme européenne a été instaurée afin de garantir la potabilité de l'eau. En fait, cette limite de $50 \mathrm{mg} / \mathrm{l}$ de nitrates (= 11,2 $\mathrm{mg}$ de N-NO3-) est désormais bien souvent largement dépassée, notamment dans le Sud-Ouest.

\section{Le site expérimental étudié.}

Le bassin-versant d'Auradé (Gers) est situé à $35 \mathrm{~km}$ à l'ouest de Toulouse (fig. 1), à la limite orientale de la Gascogne gersoise et à proximité des terrasses les plus anciennes de la Garonne. Drainé vers

(1) Martin PAEgelow, Système d'information géographique et gestion de l'environnement. Application à l'étude des sols et de la pollution par les nitrates d'origine agricole en bassin-versant expérimental. Toulouse, Université de Toulouse-Le Mirail, 1991. 2 tomcs (texte et figures), 327 p., 181 fig., 39 tabl., 156 réf., biblio. (Thèse de doctorat de géographie). 
l'ouest par le Montoussé, un affluent de la Save, ce bassin-versant s'étend sur 328 hectares. Il est inscrit dans la molasse oligo-miocène, une formation d'origine pyrénéenne dont la lithologie est très variée dans le détail.

La configuration géomorphologique, qui se résume à une succession de vallons et de collines orientés généralement du nord-ouest au sudest, permet de distinguer cinq sous bassins-versants (fig. 1). La pente moyenne est de l'ordre de $15 \%$ et avoisine par endroit $30 \%$. Les sols, très variés, issus aussi bien du substratum molassique que du manteau colluvial, sont caractéristiques de la région du Terrefort et s'ordonnent plus ou moins en toposéquences où dominent les sols bruns calcaires et calciques. Dans ces sols argileux, peu perméables, le transfert des solutés en profondeur reste limité.

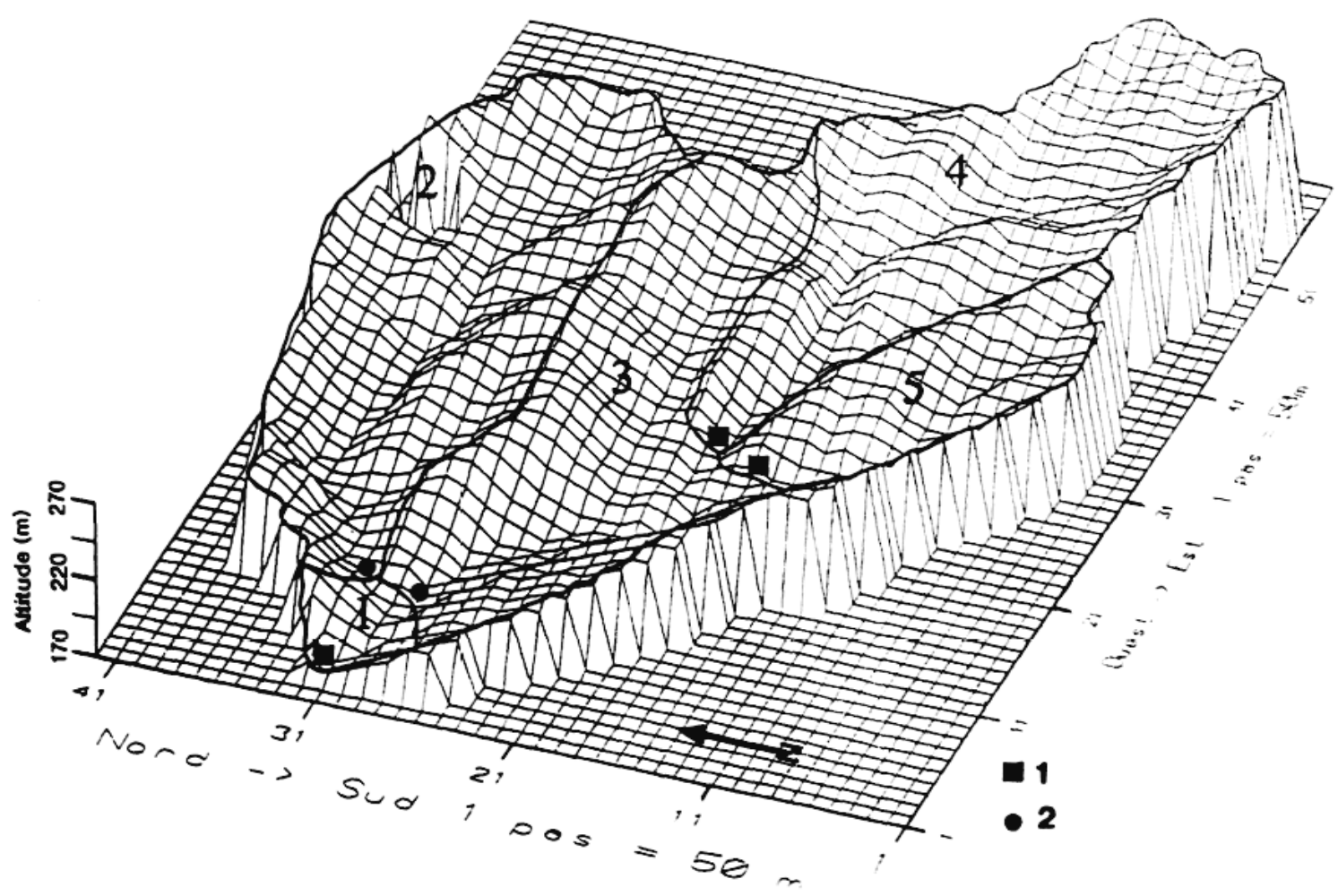

FIG. 1

Situation, subdlvisions et équipement du bassin-versant étudié à Aurad6.

Les cinq sous bassins-versants sont numérotés de 1 à 5 .

Les exutoires sont suivis en continu (1) ou chaque semaine (2).

Plus de $90 \%$ de la superficie du bassin-versant d'Auradé sont consacrés aux cultures annuelles intensives (blé tendre, tournesol, colza, blé dur, orge). L'azote est apporté sous forme d'engrais minéraux en doses variant selon l'année et l'agriculteur. A titre d'exemple, le blé tendre reçoit en moyenne $150 \mathrm{~kg} / \mathrm{N} / \mathrm{ha}$. 
Dans les eaux courantes, les concentrations nitriques (seule forme d'azote minéral présente en quantité mesurable) témoignent d'une périodicité saisonnière avec des concentrations dépassant régulièrement la norme des $50 \mathrm{mg} / 1 \mathrm{NO3}$ - au printemps (2). Concentrations nitriques et débits de ruisseau sont mesurés en continu à trois exutoires de sous bassins-versants sur cinq. Les deux autres font l'objet d'un suivi hebdomadaire (fig. 1).

\section{Conception méthodologique.}

Nos recherches se sont déroulées dans un bassin-versant expérimental : celui-ci, grâce à son compartimentage hydrologique, permet le suivi dans le temps et dans l'espace des solutés tels que le nitrate et l'établissement de bilans de transfert. La praticabilité des résultats et leur transposition opérationnelle sont facilitées par le fait que les recherches ont eu lieu sous conditions agronomiques et économiques réelles.

Au départ, les objectifs de la recherche étaient clairement définis : entre un pool d'entrée $\mathbf{N}$ (assez bien contrólé) et un flux sortant $\mathbf{N}$ mesuré aux exutoires, il s'agissait en effet d'aborder la « boîte noire " du bassin-versant selon trois ang!es complémentaires :

a) identifier, caractériser et cartographier les principales structures paysagères, de façon à mieux cerner leurs rapports avec la dynamique de l'azote minéral et à repérer, parmi ces structures, les plus favorables à la lutte contre la pollution par les nitrates;

b) mettre en place une série d'expérimentations qui permettent de quantifier les rapports entre certaines structures paysagères et la dynamique de l'azote nitrique.

c) proposer, sur ces bases, un jeu de mesures propres à réduire sensiblement la pollution nitrique, sans compromettre pour autant la conduite des exploitations ni leur compétitivité.

Cependant, pour répondre à ces objectifs, il nous a fallu résoudre deux obstacles d'ordre méthodologique.. Comme dans toute recherche sur l'environnement, la gestion des données implique, d'une part, la manipulation d'une masse considérable d'informations de nature disparate. Nous avons opté pour la mise en place d'une base de données localisées avec son système d'exploitation. Cela poussait donc à s'orienter vers la conception d'un "système d'information géographique » sur le site d'Auradé.

(2) Le bassin-versant fait l'objet depuis 1987 d'une collaboration entre le CIMA, URA 366 CNRS, (Université de Toulouse-Le Mirail) et la "Société chimique de la Grande Paroisse ", avec l'aide du Syndicat national de l'industrie des engrais, du ministère de l'Environnement ( Mission eau-nitrates ») ct de la région Midi-Pyrénées. 
Le sol, compartiment-clé situé entre les cultures et les eaux de drainage appelait nécessairement, d'autre part, une étude détaillée, compte tenu de son importance dans la dynamique de l'azote minéral et dans le transfert des nitrates. Afin d'atteindre une densité d'information pédologique élevée et cohérente avec celle des autres paramètres, physicogéographiques et agronomiques notamment, il fallait adopter une stratégie d'échantillonnage statistique propre à acquérir, au moindre coût, les données pédologiques et à déterminer leur représentation spatiale.

Ainsi, sans renoncer à nos objectifs initiaux de recherche, avons-nous été amené à les infléchir et à les replacer dans une perspective plus méthodologique, qui est celle des approches statistiques spatiales et des systèmes d'information géographique.

Nous avons choisi ici de laisser de côté tous les aspects relatifs à la constitution de la base de données et du "système d'information géographique ", ainsi que les procédures géostatistiques employées pour réaliser l'étude pédologique. En revanche, nous présentons un tableau succinct des résultats de l'étude de la dỵnamique de l'azote minéral, dont nous tirons toute une série de mesures antipolluantes, adaptées aux pays molassiques du Sud-Ouest et qui pourraient y être mises en œuvre.

\section{Dynamique de l'azote minéral et mesures antipolluantes.}

L'analyse comparative de la configuration agronomique des sous bassins-versants souligne l'incohérence apparente entre les entrées, la consommation et les résidus, d'une part, et les sorties $\mathbf{N}$ par les eaux superficielles - la molasse étant considérée comme peu perméable d'autre part. Ainsi le sous bassin-versant 5, de loin le plus polluant, reçoit-il à peine plus d'engrais azotés que les autres. Par contre, le sous bassin-versant 2, fertilisé presqu'aussi abondamment que le 5, révèle les taux de nitrate les plus modérés à l'exutoire.

L'hypothèse que des facteurs autres que ceux liés aux pratiques agronomiques dominantes interviennent dans la dynamique de l'azote minéral et produisent des effets différents dans les sous bassins-versants comparés, est corroborée par les réultats de l'interrogation de la base de données, à propos de la configuration physico-géographique des sous bassins-versants. Celle-ci, en effet, est sensiblement contrastée en ce qui concerne l'étendue des sols hydromorphes et l'aménagement de bas de pentes et des talwegs. Ainsi les sous bassins-versants à rejets nitriques modérés ont-ils un taux de végétation pérenne (bois et prairies) sur sites hydromorphes de bas de versant et de talwegs supérieur à la moyenne et vice-versa.

Ainsi se profile le rôle des facteurs liés, dans la dynamique de l'azote nitrique, à l'agencement interñ des unités de terroir. Ce rôle a été 
précisé à partir d'une série de mesures et d'expérimentations par bougies poreuses dans des zones dites sensibles.

Ces zones sensibles correspondent à des sites où le cycle de l'eau est ralenti et où le confinement induit des conditions hydromorphes propices à la dénitrification. Leur position intercalée entre les cultures et les axes de drainage superficiel, ainsi que leur faible étendue, leur confèrent un rôle stratégique dans la lutte contre la pollution nitrique.

Un certain nombre d'éléments paysagers traditionnels, situés en zones sensibles, ont été testés quant à leur effet sur la dynamique de l'azote minéral et l'éluviation des nitrates en provenance des cultures vers les eaux de drainage superficiel: prairies, haies, type d'aménagement de berges (boisé ou à une seule strate végétale herbacée), mouillères de versant. Bien que ces suivis nécessitent une itération, ils confirment l'importance de l'aménagement des bas de versant et des talwegs. Un complexe haie-prairie en situation de fond de val!on, par exemple, réduit sensiblement les concentrations nitriques par rapport aux champs à cultures annuelles environnantes (d'un facteur 10 à 30 ). Les concentrations nitriques sous berge boisée sont nettement inférieures à celles enregistrées sous berge ayant une seule strate végétale herbacée. Enfin, le fonctionnement des mouillères de versant illustre, à échelle réduite, le mécanisme des fonds de bassin entier: l'extrême hydromorphie (g'eyification superficielle) induit des conditions d'anaérobiose propice à la dénitrification.

Suite à ces résultats encourageants, un enherbement partiel (3) des bords de ruisseau d'un sous bassin-versant, jusqu'alors complètement dénudé, a été entrepris pour connaître la largeur minimale et le temps d'installation d'une telle mesure. On a démontré l'effet de l'enherbement en exprimant les concentrations nitriques mesurées au bord du ruisseau à l'aval d'une bande enherbée large de $12 \mathrm{~m}$, à 30,60 et $90 \mathrm{~cm}$ de profondeur, en pourcentages de celles enregistrées à l'amont immédiat de cette bande (limite entre culture et bande d'enherbement) (fig. 2). Les concentrations profitant de la zone tampon ne dépassent jamais $40 \%$ de celles mesurées à la limite du champ; généralement, elles se situent en deçà de $10 \%$.

L'ensemble des expérimentations conduit à démontrer qu'un aménagement agro-paysager tirant parti des sites à tendance naturelle hydromorphe, exerce un contrôle efficace sur la dynamique de l'azote minéral. Les éléments paysagers analysés sont des pièges naturels pour l'azote. Sur ces bases, un lot de mesures antipolluantes localisées, peu contraigantes, adaptées au contexte régional et tirant profit de dix années d'expérience vécue dans le cadre du bassin-versant d'Auradé, a été pro-

(3) Les essais menés, notamment celui portant sur l'enherbement n'ont été possibles, il convient de le rappeler, que grâce à la bienveillance et à l'esprit de coopération active des agriculteurs d'Auradé (Gers), qui ont consenti à convertir un tiers d'hectare de terres arables en prairie de fauche. 
posée. Nous entendons par " localisé » le fait qu'une mesure s'applique à un espace défini et délimité faisant ainsi référence au zonage et à l'aménagement des terroirs. Ces propositions se veulent complémentaires aux mesures non localisées classiques portant sur les pratique culturales (doses d'engrais, date d'apport, enfouissement des pailles, etc.).

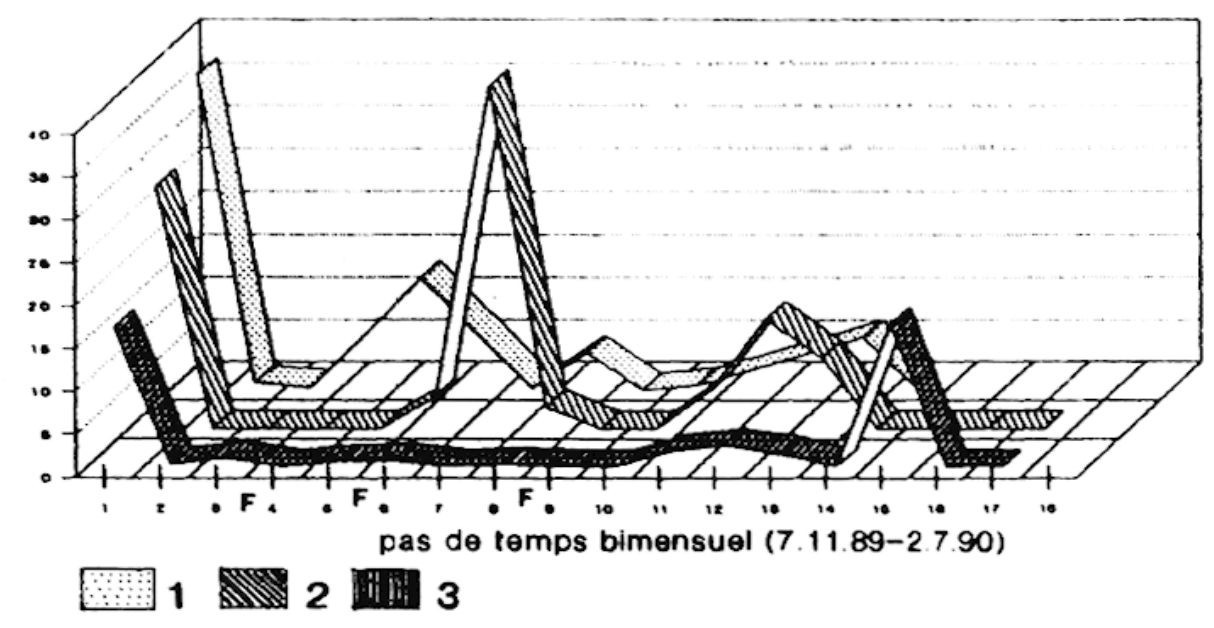

FIG. 2

Effet d'un enherbement large de $12 \mathrm{~m}$ (sous bassin-versant no 5 ).

Mesure en pourcentage des différences de concentration de N-NO3 du lot 4. Profondeur: $1.90 \mathrm{~cm} .-2.60 \mathrm{~cm} .-3.30 \mathrm{~cm}$.

Cinq ensembles de mesures ont été proposés :

a. Enherbement des bords de cours d'eau et maintien d'une végétation pérenne en zone hydromorphe (largeur $12 \mathrm{~m}$ );

b. Diminution des apports azotés d'un tiers sur les zones de contact entre enherbement et cultures (largeur $12 \mathrm{~m}$ );

c. Réalisation de travaux aratoires perpendiculaires à la pente sur les bas de versant en pente faible;

d. Insertion de petites mares sur les axes de drainage superficiel de chaque sous bassin-versant;

e. Implantation de haies arbustives sur les limites de parcelles agricoles perpendiculaires aux bas de versant en pente forte.

Les propositions visant un changement d'affectation ou de gestion des terroirs ne touchent que $3,1 \%$ de la surface du bassin-versant. Quant à la réduction des apports azotés, elle ne porte que sur $4,3 \%$ de la surface d'Auradé.

Praticabilité et efficacité des mesures proposées et validées ont été vérifiées dans un sous bassin-versant test où sont réalisées depuis 1990 
les propositions $a, b$ et $c$. Il est encore trop tôt pour en tirrer des conclusions fondées, mais les premiers résultats sont très prometteurs.

Ces recherches, comme toute recherche dans le domaine de l'environnement, n'ont donc de sens que pour autant qu'une certaine continuité leur est garantie. C'est dans cette optique que le bassin-versant d'Auradé, suivi depuis le début des années 1980, prend toute sa valeur et est en passe de devenir un observatoire permanent pour l'environnement.

Enfin, la mise en relation systématique de la dynamique de l'azote minéral avec les paramètres descripteurs du milieu, n'aurait pu être menée de façon aussi aisée sans la mise en place d'une base de données localisées et les traitements géostatistiques qu'elle permet. L'établissement et l'exploitation de systèmes d'information géographique adaptés et spécifiques, est devenu aujourd'hui un impératif pour l'analyse et la gestion de l'environnement.

Martin PaEgelow.

\section{PAYSAGES DE LA HAUTE GARONNE DEPUIS LES TEMPS GLACIAIRES}

La dynamique du paléoenvironnement du bassin supérieur de la Garonne, a été établie depuis la fin des temps glaciaires, jusqu'à l'Actuel, en utilisant les principes d'une approche transdiciplinaire systémique (4).

\section{Formations glaciaires et formations lacustres.}

Les formations morainiques $d u$ "glaciaire ancien 》 du bassin glaciaire terminal de la Garonne, appartiennent à un ou plusieurs épisodes glaciaires antérieurs au Wurm. Les forrnations morainiques du "glaciaire récent "sont rattachées à la glaciation wurmienne. Elles traduisent: a) un épisode d'avancée maximale des glaces (Burs - Coumeaux); b) une première phase de retrait (La Serre) ; c) une ultime mais longue période de décrue dont le déclenchement est antérieur au Wurm final.

Trois sondages effectués dans le lac de Barbazan, ont permis de reconstituer la dynamique de sédimentation dans ce site, depuis le Pléniwurm. Pendant la déglaciation wurmienne, le lac de Barbazan était un lac de

(4) Valérie ANDRIEU, Dynamique du paléoenvironnement de la vallée montagnarde de la Garonne (Pyrénées centrales, France) de la fin des temps glaciaires à l'Actuel. Toulouse, Université de Toulouse-Le Mirail, 330 p., 86 fig., 17 tabl., 12 fig. h.t., 1 tabl., glossaire, index, 380 réf., biblio. 